\title{
Ray optics hamiltonian approach to relativistic self focusing of ultraintense lasers in underdense plasmas
}

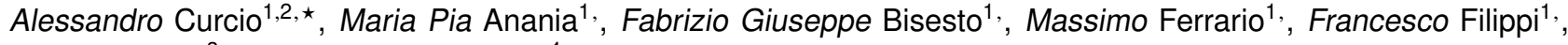 \\ Danilo Giulietti ${ }^{3}$, and Massimo Petrarca ${ }^{4}$, \\ ${ }^{1}$ INFN-LNF, via Enrico Fermi 40, 00044 Frascati, Rome, Italy \\ ${ }^{2}$ Department of Physics, "Sapienza" University of Rome, Piazzale A. Moro 2, I-00185 Rome, Italy \\ ${ }^{3}$ Physics Department of the University and INFN, Largo Bruno Pontecorvo 3, 56127 Pisa, Italy \\ ${ }^{4}$ Department of Basic and Applied Sciences for Engineering (SBAI) and INFN-Roma1, "Sapienza" University of Rome, Via A. \\ Scarpa 14, 00161 Rome, Italy
}

\begin{abstract}
The relativistic self focusing of an ultraintense laser propagating through an underdense plasma is analyzed from a geometrical optics point of view, exploiting the classical hamiltonian formalism. The distribution of the laser intensity along the self-generated plasma channel is studied and compared to measurements.
\end{abstract}

\section{Introduction}

The relativistic self focusing of ultraintense lasers in underdense plasmas is a well-known phenomenon, of great interest in last decades after the advent of ultrashort lasers of the femtosecond-class. Self focusing effects have been observed since the first era of pulsed laser systems of nanosecond-class, but only with new compression techniques (Chirped Pulse Amplification [1]) and consequently with the possibility to exploit ultrashort laser pulses it became possible to experimentally explore the variety of optical phenomena which relate to relativistic optics [2]. The relativistic optics is the study of the optical response of the matter when the electron quiver velocity $v_{q}=a_{0} c / \sqrt{1+a_{0}^{2}}$ under the action of an ultraintense electromagnetic field reaches values close to the velocity of light in vacuum $c$. The relativistic parameter $a_{0}=8.5 \times 10^{-10} \lambda_{0}[\mu \mathrm{m}] \sqrt{I_{0}\left[\mathrm{~W} / \mathrm{cm}^{2}\right]}$ related to the laser wavelength $\lambda_{0}$ and intensity $I_{0}$, allows to define the threshold above which an optical phenomenon can be classified as relativistic, i.e. $a_{0} \gtrsim 1$. The relativistic self focusing is actually a relativistic phenomenon typical for ultraintense laser pulses propagating through underdense plasmas [36]. In fact the Lorentz factor of a free electron quivering under the action of a linearly polarized laser pulse is given by $\gamma=\sqrt{1+a^{2} / 2}$ [7]. In reality the laser beam has a finite and well-defined radial profile, therefore, in a fluid picture of the plasma electrons, the factor $\gamma(r)$ is a function of the radial coordinate $r$ via the laser intensity profile, contained in $a$. For sake of simplicity we are limiting our considerations to a radial-symmetric physical system. In most of practical and realistic cases the laser intensity profile is peaked in $r=0$ and decreases for $r>0$.

\footnotetext{
^e-mail: alessandro.curcio@uniroma1.it
}

It means that the on-axis plasma electrons are "heavier" than the off-axis electrons if looking at the Lorentz factor $\gamma(r)$. The index of refraction for a plasma medium is generally $n=\sqrt{1-\omega_{p}^{2} / \gamma(r) \omega_{0}^{2}}$, where $\omega_{p}=\sqrt{n_{e}} e^{2} / m \varepsilon_{0}$ is the plasma frequency and $\omega_{0}=2 \pi c / \lambda_{0}$ is the laser pulsation. The plasma frequency is defined through the elementary charge $e$, the electron plasma density $n_{e}$, the dielectric constant of vacuum $\varepsilon_{0}$ and the electron mass $m$. The $r$ dependence of the Lorentz factor $\gamma$ in the expression of the plasma index of refraction is the cause for relativistic self focusing. In fact the plasma electron profile at the laser front acts like a focusing lens. This focusing effect can be effective as far as the laser intensity is maintained so high that relativistic quiver velocities are provided to the plasma electrons at the pulse front. The relativistic self focusing is responsible in guiding laser beams giving rise to typical laser envelope oscillations, manifesting the counterbalance of focusing/defocusing effects. We can find an optimum condition for the guiding of the laser in the linear and slightly non-linear regime of laser-plasma interaction, by equating the laser electric field to the limit field $E_{w b}$ (also called wavebreaking field [7]), then supposing to have a diffraction-limited beam with cross-section $\sim \lambda_{0}^{2}$. The power associated to this beam is defined as critical power for relativistic self focusing $P_{c r}$ :

$$
P_{c r} \sim I_{w b} \lambda_{0}^{2}=c \frac{\varepsilon_{0}}{2} E_{w b}^{2} \lambda_{0}^{2} \sim 14\left(\frac{\omega_{0}^{2}}{\omega_{p 0}^{2}}\right) G W
$$

where the wavebreaking intensity $I_{w b}$ is the intensity of an electromagetic wave with electric field $E_{w b}$, i.e. $I_{w b}=$ $c \varepsilon_{0} E_{w b}^{2} / 2$. A more precise and more complex calculation with an undulatory description of the laser through a nonlinear Schroedinger equation would give a factor 17.5 in place of 14 [8], nevertheless our naive calculation clearly 
enlightens the physics of the laser guiding process, as a counterbalance between the laser and the plasma electric fields. In particular for power exceeding $P_{c r}$ self focusing can occur, otherwise diffraction dominates. This is due to the fact that the laser field is not effective in shaping the electron density into such a profile useful for the guiding until the laser electric field reaches the maximum plasma electric field $E_{w b}$. In fact below this threshold the electrons move under the action of the plasma electric field, being the laser action only a small correction to their dynamics. When the pump is depleted, i.e. when the laser energy has been exhausted in the vastness of interaction processes which couple the laser energy to the plasma electrons (in particular to Langmuir waves ), a net defocusing overcomes. Efficient relativistic self focusing has been experimentally demonstrated, so far that the laser propagation at high-intensity has been observed over paths greater than tens of Rayleigh lengths. In the present letter we introduce a ray optics hamiltonian approach [9] to the propagation of a ultraintese laser through an underdense plasma, with the main aim to study the effects of relativistic self focusing. Other fundamental works have been done in the past about the relativistic self focusing, all based on non linear wave-optics, eventually recalling a hamiltonian formalism related to non-linear Schroedinger equations. Starting from the consideration that the ratio $\lambda_{0} / \lambda_{p}<<1$ in typical cases of laser-plasma interaction, where $\lambda_{p}=2 \pi c / \omega_{p}$ is the plasma wavelength, we develop a different approach to the physical problem under study, based on ray-optics instead than wave-optics, in particular we are going to make use of hamiltonian optics. In fact the parameter $\lambda_{0} / \lambda_{p}$ can set the border between a undulatory description with respect to a geometrical description of the laser light propagating through an underdense plasma, indeed when also the condition $\sigma>>\lambda_{0}$ is satisfied, where $\sigma$ is the beam size parameter. The typical length scale to compare in this case to the laser wavelength in order to determine if a wave optics approach is needed is the plasma wavelength. For $\lambda_{0} / \lambda_{p}<<1$ a geometrical approach is justified. We show that, even with some limitations in the description of laser dynamics which nevertheless don't compete to the Relativistic Self Focusing (RSF) effect, a simple model can be built in order to study the RSF based on geometric, hamiltonian optics. We are going to study the trajectory of the light rays throughout an underdense plasma in the condition for RSF to occur. In the end a beam envelope trend is obtained, after the reconstruction of the laser beam starting from the single ray components. Computationally speaking, this approach turns out to be very fast, suitable for quick physical estimations which PIC codes, even if more complete and exhaustive in the physical description of phenomena, are not able to provide.

\section{Theoretical background}

The hamiltonian for a light ray of a light beam propagating in the $z$ direction through a medium characterized by an index of refraction $n(r, z)$ is given by:

$$
H=-\sqrt{n^{2}-p_{r}^{2}}
$$

where $p_{r}$ is the light ray radial momentum. The Hamilton equation for the divergence $\theta=d r / d z$ is:

$$
\frac{\partial H}{\partial p_{r}}=\theta=\frac{p_{r}}{\sqrt{n^{2}-p_{r}^{2}}}
$$

from which the useful relation is obtained:

$$
p_{r}=\frac{n \theta}{\sqrt{1+\theta^{2}}}
$$

where we used equations 2 and 3 to get the result 4 . The second Hamilton equation gives:

$$
\frac{d p_{r}}{d z}=\frac{n}{\sqrt{n^{2}-p_{r}^{2}}} \frac{d n}{d r}=\frac{n \theta}{p_{r}} \frac{d n}{d r}=\sqrt{1+\theta^{2}} \frac{d n}{d r}
$$

By deriving equation 3 by $z$ (under the assumption $d \theta / d z>>d n / d z$, corresponding to the slowly varying envelope approximation for the laser) and equating to equation 4 , an equation for the only variable $\theta$ is obtained:

$$
\frac{d \theta}{d z} \approx\left(1+\theta^{2}\right)^{2} \frac{d \log n}{d r}
$$

The radial coordinate of the light ray as function of $z$ comes directly from the integration of $\theta$ after solving the equation 5, namely:

$$
r(z)=r_{0}+\int_{0}^{z} \theta d z
$$

where $r_{0}$ is the initial radial position of the light ray. Notice that equation 5 is analytically integrable, yielding the following result for $\theta$ :

$$
\frac{1}{2}\left(\frac{\theta}{1+\theta^{2}}+\arctan \theta\right)=\Theta+\int_{0}^{z} \frac{d \log n}{d r} d z
$$

where $\Theta$ is an integration constant. When considering for example a laser beam it is possible to define, once the radial distribution $\mathrm{R}(\mathrm{r})$ of the laser energy is known, the beam radius:

$$
\sigma(z)=\sqrt{<r\left(z, r_{0}\right)^{2}>-<r\left(z, r_{0}\right)>^{2}}
$$

where we defined the average on the radial distribution of the laser beam energy as:

$$
<f\left(r, r_{0}\right)>=\frac{\int_{0}^{\infty} d r_{0} R\left(r_{0}\right) f\left(r, r_{0}\right)}{\int_{0}^{\infty} d r_{0} R\left(r_{0}\right)}
$$

For what concerns the index of refraction of the underdense plasma we consider here, we make the following assumptions: the regime of the acceleration is highly nonlinear, in particular we refer to the bubble regime, the electron plasma density at the front of the laser pulse is assumed unmodified during the propagation. We neglect longitudinal non-linear effects like steepening of the front pulse, only retaining in an empiric way the effect of the pump depletion. These choices are motivated by the fact 
that the main concern here is to study the transverse dynamics of the laser beam under consideration which undergoes relativistic self-focusing. The expression of the laser normalized potential we take into account is:

$$
a=\frac{a_{0}}{\sqrt{2}} \exp \left(-\frac{r^{2}}{\sigma_{0}^{2}}-\frac{z}{2 L_{p d}}\right)
$$

where the pump depletion length is $L_{p d}=c \tau \omega_{p}^{2} / \omega_{0}^{2}$ and $R\left(r_{0}\right)=\exp \left(-2 r^{2} / \sigma_{0}^{2}\right)$ [10]. The laser pulse duration is $\tau$ and the initial laser beam size parameter is $\sigma_{0}$.

\section{Comparison with current experiments}

In the following we develop a calculation referring to an experiment of Laser Wakefield Acceleration (LWFA) performed at the SPARC-LAB test facility through the interaction of the ultrashort ultraintense $T i: S a$ laser FLAME with a $\mathrm{He}$ gas-jet target $[11,12]$. The initial normalized vector potential relative to the experiment was $a_{0} \sim 4$, corresponding to a laser pulse of energy $\lesssim 1 J$, duration $\tau=30 \mathrm{fs}$ and focal spot radius $6 \pm 1 \mu \mathrm{m}$, equivalent to $\sigma_{0}=8.5 \mu \mathrm{m}$ in Eq. 10. A single-shot set of diagnostic were adopted to determine the extension of the plasma generated by the laser pulse and the relative plasma electron density. In particular a Mach-Zender interferometer was installed in order to retrieve the electron plasma density value. The result of the measurement was $n_{e} \sim 8 \times 10^{18} \mathrm{~cm}^{-3}$. In Fig. 1 the interferogram of the laser-produced plasma channel due to the propagation of the ultraintense laser FLAME through the supersonic $\mathrm{He}$ gas-jet, from left to right, is reported. The resolution of the image was $5 \mu \mathrm{m}$ per pixel. Once the laser and plasma

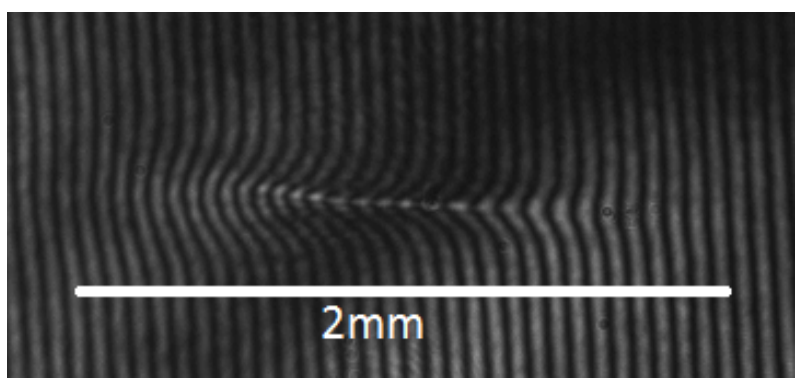

Figure 1. The interferogram of the laser-produced plasma channel due to the propagation of the ultraintense laser FLAME through the supersonic He gas-jet, from left to right.

parameters are known it becomes possible to exploit the model developed above for evaluations regarding relativistic self focusing, in order to verify the validity of the model itself in comparison to the experimental data. The first calculation we show is reported in Fig. 2 where the expected trend of the laser beam radius $\sigma$ in vacuum is compared to the one obtained by virtue of the relativistic self focusing, when the ultraintense laser pulse FLAME propagates through the underdense He plasma inducing relativistic electron quivering. The beam radius in presence of the plasma fluctuates while it is guided by RSF. After a depletion length $L_{p d} \sim 1.9 \mathrm{~mm}$ the laser radius starts to increases because of the upper hand of the defocusing. The region of high laser intensity is slightly more than one millimeter which is almost ten times the Rayleigh length corresponding to the considered laser beam. The situation is more evident if looking at Fig. 3 where the normalized laser intensity $a^{2}$ is represented. The laser intensity is reconstructed by summing up the contributions of each light ray: the local density of the light rays is proportional to the laser local intensity. The imposition of the initial condition 10 ensures a self-consistent way to quantitatively trace the laser intensity along the plasma channel. The calculated extension of the laser high-intensity region is reasonably comparable to the extension of the high electron plasma density region of Fig. 1, recognizable as the region with more-pronounced curvature of the interferometric lines. In effect a laser high-intensity region must correspond to high electron plasma density by virtue of the local ionization efficiency, which increases with the local photon number.

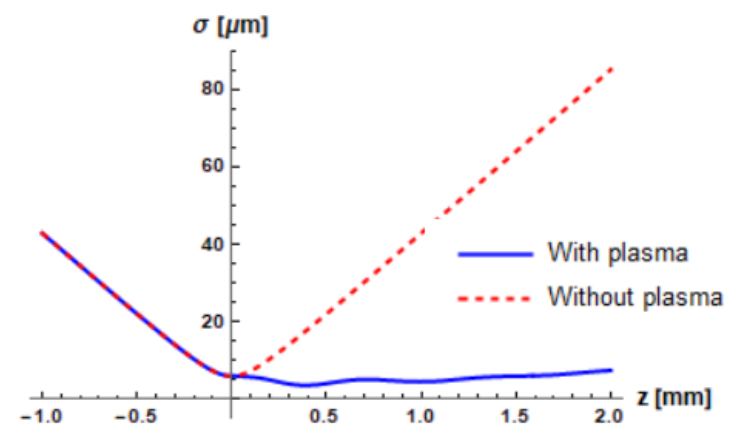

Figure 2. The comparison between the free propagation beam radius of the FLAME laser in vacuum (red) and the beam radius determined by the relativistic self focusing of FLAME propagating inside the He underdense plasma (blue). The zero point corresponds to the beginning of the plasma region $(z>0)$.

In fact the optical field ionization (OFI) is a multiphotonic process, for which the probability of tunneling ionization of a bound electron increases with the local laser intensity. Fig. 3 contains even another important information regarding the distribution of the laser energy along the plasma channel, very high in the beginning of the interaction and then slowly decreasing. This trend which we can observe in our calculation is in effect a physical feature we can observe even in Fig. 4. In fact a second diagnostics for the laser propagation inside the He gas-jet was adopted, based on optical Thomson scattering [13]. In this process the laser light is scattered by the plasma electrons escaping from the bound atomic states with a residual kinetic energy, and consequently it is Doppler-shifted in a way correlated to the electron velocities. Fig. 4 shows a RGB picture of the plasma channel generated by the laser propagating in the He gas. The colors are related to the local electron plasma temperature and also to broadening of the laser spectrum due to Self-Phase Modulation [14], which in turn is proportional to the local laser intensity. The plasma channel length is comparable to the length cal- 


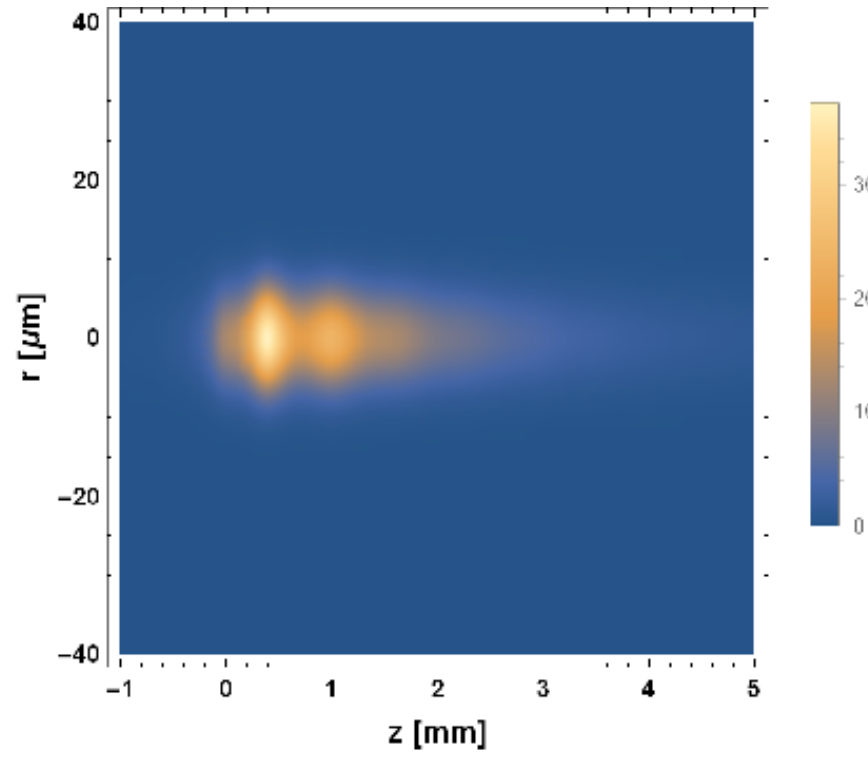

Figure 3. Normalized laser intensity $a^{2}$ of the laser FLAME propagating from left to the right from vacuum to the He gas-jet. The zero point corresponds to the beginning of the plasma region $(z>0)$.

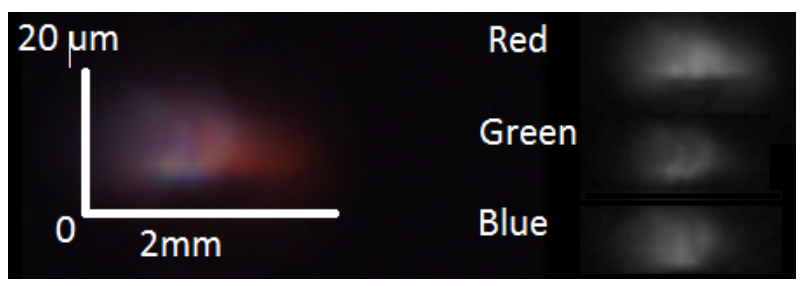

Figure 4. Picture of the plasma channel in RGB colors: red $(570-750 \mathrm{~nm})$, green $(495-570 \mathrm{~nm})$, blue $(380-495 \mathrm{~nm})$.

culated from Fig. 3, in particular the length corresponding to the blue light in Fig. 4 is comparable to the length of maximum intensity of the laser calculated from Fig. 3, which is about $1.2 \mathrm{~mm}$. The ray optics model for relativistic self focusing can reproduce the extension of the region of the maximum laser intensity, which is an useful benchmark for laser plasma acceleration experiments where the knowledge of the laser maximum intensity region extension allows the determination of the most suitable plasma density for the acceleration. In fact the dephasing length for the accelerating electrons, i.e. the length after which the electrons start to experience a decelerating field, it is strongly determined by the plasma density and it is of fundamental importance to know to what extent the laser can propagate at high intensity producing wakefields that accelerate electrons with the maximum efficiency.

\section{Conclusions}

We have presented a model based on hamiltonian ray optics capable of reproducing experimental results related to relativistic self focusing phenomena. The ratio $\lambda_{0} / \lambda_{p}$ determines the limit between the geometrical and wave optics. An underdense plasma, namely a plasma in which a laser pulse can propagate, always satisfies the condition $\lambda_{p}>>\lambda_{0}$, therefore a geometrical optics description of phenomena like the relativistic self focusing is possible and justifiable. The comparison with experimental data confirm this assumption.

\section{References}

[1] D. Strickland and G. Mourou, "Compression of amplified chirped optical pulses”, Opt. Commun. 56, 219 (1985).

[2] Borovsky, Andrew V., et al. Laser physics at relativistic intensities. Vol. 34. Springer Science Business Media, 2013.

[3] Askar'yan, G. A. (1962). "Cerenkov Radiation and Transition Radiation from Electromagnetic Waves". Journal of Experimental and Theoretical Physics. 15 (5): 943-6.

[4] Max, Claire Ellen, Jonathan Arons, and A. Bruce Langdon. "Self-modulation and self-focusing of electromagnetic waves in plasmas." Physical Review Letters 33.4 (1974): 209.

[5] Hora, Heinrich. "Theory of relativistic self-focusing of laser radiation in plasmas." JOSA 65.8 (1975): 882886.

[6] Feit, M. D., A. M. Komashko, and A. M. Rubenchik. "Relativistic self-focusing in underdense plasma." Physica D: Nonlinear Phenomena 152 (2001): 705713.

[7] Esarey, E., C. B. Schroeder, and W. P. Leemans. "Physics of laser-driven plasma-based electron accelerators." Reviews of Modern Physics 81.3 (2009): 1229.

[8] Mourou, Gerard A., Toshiki Tajima, and Sergei V. Bulanov. "Optics in the relativistic regime." Reviews of modern physics 78.2 (2006): 309.

[9] Buchdahl, Hans Adolph. An introduction to Hamiltonian optics. Courier Corporation, 1993.

[10] Lu, Wei, et al. "Generating multi-GeV electron bunches using single stage laser wakefield acceleration in a 3D nonlinear regime." Physical Review Special Topics-Accelerators and Beams 10.6 (2007): 061301.

[11] Curcio, A., et al. "Trace-space reconstruction of lowemittance electron beams through betatron radiation in laser-plasma accelerators." Physical Review Accelerators and Beams 20.1 (2017): 012801.

[12] Curcio, A., et al. "Single-shot non-intercepting profile monitor of plasma-accelerated electron beams with nanometric resolution." Applied Physics Letters 111.13 (2017): 133105.

[13] Hutchinson, I.H. (2005). Principles of Plasma Diagnostics. Cambridge University Press.

[14] Giulietti, D. and Curcio, A. Self-Phase Modulation Eects as Laser Produced Plasma Diagnostics. Journal of Instrumentation 11(08):C08011-C08011 August 2016 DOI: 10.1088/1748-0221/11/08/C08011 